\title{
INTERPRETATION OF TRAUMATIC LUMBAR PUNCTURE
}

The records of two cohorts of children who received lumbar puncture (LP) for suspected meningitis at Children's Memorial Hospital, Chicago, IL, were reviewed retrospectively to determine the value of a ratio of observed to predicted $(\mathrm{O}: \mathrm{P})$ cerebrospinal fluid (CSF) white blood cells (WBC) after a traumatic LP in the differentiation of children with and without meningitis. The predicted CSF WBC count was calculated using the formula CSF RBC x blood WBC/blood RBC (blood counts had been obtained within 6 hours of LP). All 57 children in the study were older than 1 month and the CSF had $>500 / \mathrm{mm}^{3}$ red blood cells (RBC). Cohort 1 , consisting of $12(21 \%)$ patients examined in 1990 through 1999, had CSF cultures positive for a bacterial pathogen. Cohort 2 patients $(\mathrm{n}=45[79 \%])$ were tested during Jan-Dec 1999, in the post- $H$ influenzae $\mathrm{b}$ vaccine era, and had a CSF culture negative for bacterial pathogens. Patients receiving antibiotics within 72 hours before LP, and those with a previous neurosurgical procedure or CNS bleed were excluded.

Patients with meningitis were significantly older (median 7.8 months; range 1-106 months) than those without meningitis (median 1.3 months; range 1-139 months). The O:P and $\mathrm{WBC}: \mathrm{RBC}$ ratios were significantly lower for patients without, compared to those with meningitis (medians 0.064 and 0.001 of 1.26 and 1.98 , respectively). An O:P ratio $<0.01$ and WBC:RBC ratio $<1: 100(0.01)$ were each $100 \%$ predictive of the absence of meningitis. The absence of pleocytosis was also highly specific for a negative culture, whereas a negative Gram stain, lack of polymorphonuclear cell predominance, CSF glucose, and protein were less predictive. When the LP is traumatic, both the WBC:RBC ratio and the O:P ratio may be helpful in the identification of patients without meningitis, but the former calculation is less cumbersome and is recommended for an initial screening. All clinical and laboratory data should be evaluated before deciding not to treat for meningitis after a traumatic tap. (Mazor SS, McNulty JE, Roosevelt GE. Interpretation of traumatic lumbar punctures: Who can go home? Pediatrics March 2003;111:525-528). (Reprints: Suzan S Mazor MD, 2300 Children's Plaza, Box 62, Chicago, IL 60614).

COMMENT. Traumatic lumbar punctures (LP) are difficult to interpret, and apart from CSF culture, methods used to differentiate patients with or without meningitis must be viewed with caution. The CSF leukocyte count in bacterial meningitis is usually greater than $1000 / \mathrm{mm}^{3}$. CSF becomes turbid when the leukocyte count exceeds $200-400 / \mathrm{mm}^{3}$. A CSF leukocyte count of less than $250 \mathrm{~mm}^{3}$ may be present in $20 \%$ of patients with acute bacterial meningitis, and pleocytosis may be absent in patients with severe sepsis and meningitis. A traumatic LP will alter the CSF leukocyte count and protein concentration, while the gram stain, culture and glucose level may not be influenced. (Prober CG. CNS Infections. In Behrman RE, Kliegman RM, Jenson HB, Eds. Nelson Textbook of Pediatrics, $16^{\text {th }}$ edition. Philadelphia, WB Saunders Comp, 2000).

Methods proposed to correct for the presence of red blood cells have previously focused on a test using the observed:predicted (O:P) ratio of CSF WBCs for the diagnosis of meningitis. The present investigators were interested in a highly specific test and the identification of patients without meningitis by a method that would not overlook a meningitis case. They conclude that in children older than 1 month with a traumatic LP, the WBC:RBC and O:P ratios will differentiate the majority of patients without meningitis 
from those with meningitis. However, they find it prudent to advise reliance on all clinical and laboratory information, which would include bacteriologic results, before discharging a child without treatment after a traumatic LP.

\section{DEMYELINATING DISORDERS}

\section{PROGNOSIS OF RELAPSING NEUROMYELITIS OPTICA}

Clinical predictors of a relapsing course and subsequent survival in 80 patients with neuromyelitis optica (NMO) (57 relapsing, 23 monophasic) were developed from a study of medical records and patients at the Mayo Clinic, Scottsdale, AZ. Patients with monophasic disease were followed for a median of 14 years (range 1-45 years) with no relapse; median follow-up in the relapsing group was 60.2 months (range 4-372 months). Forty six $(81 \%)$ of 57 patients in the relapsing group were women compared to 11 (48\%) of 23 in the monophasic group $(\mathrm{p}=0.003)$. The median age at onset was greater ( 41 (range 6-72) vs 29 (range 1-54) years; $p=0.008$ ) and autoimmune disease was more common $(19 / 57$ vs $1 / 23 ; p=0.007)$ in the relapsing group. Female sex, older age of disease onset, milder initial impairment score, and longer time between $1^{\text {st }}$ and $2^{\text {nd }}$ attacks predict future relapsing disease ( $82 \%$ sensitivity and $74 \%$ specificity). Mortality due to relapsing NMO was correlated with a history of other autoimmune disease, higher attack frequency during the first 2 years of disease, and better motor recovery following the index myelitis event. The clinical features identified were available at diagnosis or early in the disease course, and were predictive of relapsing disease and survival. The identification of patients at high risk for severe, relapsing NMO permits early initiation of therapy to prevent relapse. (Wingerchuk DM, Weinshenker BG. Neuromyelitis optica: Clinical predictors of a relapsing course and survival. Neurology March (1 of 2) 2003;60:848-853). (Reprints: Dr Dean M Wingerchuk, Department of Neurology, Mayo Clinic, 13400 East Shea Blvd, Scottsdale, AZ 85259).

COMMENT. New NMO diagnostic criteria that exclude cases of typical multiple sclerosis, as proposed by the authors, are as follows: absolute criteria are optic neuritis, acute myelitis, and absence of disease outside the optic nerve or spinal cord: supportive criteria are major (negative brain MRI, $>3$ abnormal vertebral segments on spinal cord MRI, and CSF pleocytosis of $>50 \mathrm{WBC} / \mathrm{mm}^{3}$ or $>5$ neutrophils $/ \mathrm{mm}^{3}$ ); and minor (bilateral optic neuritis, impaired visual acuity (20/200) in at least one eye, and severe weakness in 1 or more limbs). Diagnosis requires all absolute and one major or 2 minor supportive criteria. Relapsing NMO has a poor prognosis and causes irreversible impairment. Early diagnosis by the clinical predictors identified, and differentiation of relapsing from monophasic cases of NMO, should permit early intervention and greater survival rate.

MRI and neuropathology of acute hemorrhagic leukoencephalitis (AHL) and differentiation from acute demyelinating encephalomyelitis (ADEM) are discussed in a case report of a 19-year-old man studied at the University of Buffalo, NY (Kuperan S et al. Neurology Feb (2 of 2) 2003;60:721). AHL is a hyperacute form of ADEM. MRI white matter lesions of AHL are larger with more edema than seen in ADEM, the basal ganglia are usually spared, and infiltrates are predominantly neutrophilic rather than lymphocytic 